\title{
Experimental and numerical in-plane displacement fields for determine the J-integral on a PMMA cracked specimen
}

\author{
S. Hedan ${ }^{1, a}$, V. Valle ${ }^{2}$, and M. Cottron ${ }^{2}$ \\ ${ }^{1}$ Université de Poitiers, CNRS UMR6269, Laboratoire Hydrasa, ESIP, 40 Av. du Recteur Pineau \\ 86000 Poitiers, France \\ ${ }^{2}$ Université de Poitiers, CNRS UPR3346, Institut PPRIME, SP2MI 2 Bd Marie et Pierre Curie \\ 86962 Futuroscope Chasseneuil, France
}

\begin{abstract}
Contrary to $J$-integral values calculated from the 2D numerical model, calculated Jintegrals [1] in the 3D numerical and 3D experimental cases are not very close with $J$-integral used in the literature. We can note a problem of structure which allows three-dimensional effects surrounding the crack tip to be seen. The aim of this paper is to determine the zone where the Jintegral formulation of the literature is sufficient to estimate the energy release rate $(G)$ for the $3 D$ cracked structure. For that, a numerical model based on the finite element method and an experimental setup are used. A grid method is adapted to experimentally determine the in-plane displacement fields around a crack tip in a Single-Edge-Notch (SEN) tensile polymer (PMMA) specimen. This indirect method composed of experimental in-plane displacement fields and of 2 theoretical formulations, allows the experimental $J$-integral on the free-surface to be determined and the results obtaining by the 3D numerical simulations to be confirmed.
\end{abstract}

\section{Introduction}

In linear elastic case, experimental studies using the shadow method [2], interferometric methods [3] or numerical simulations [4] mention the presence of a 3D area around the singular zone where the behaviour is three-dimensional. But, the $2 \mathrm{D}$ theory of a cracked specimen is developed with some hypotheses [5], as infinite medium and plane problem. Consequently, the stress intensity factor $\mathrm{K}$ and the $J$-integral, parameters considered constant by two-dimensional theories cannot be correctly evaluated by $3 \mathrm{D}$ numerical or 3D experimental approaches in this region. A relation between these 2 latter parameters (eq.1) exists for 2D linear elastic problems. In the literature [6], the experimental $J$ integral present a dependence $(\mathrm{R}=\mathrm{r} / \mathrm{h}<0.5)$ with the contour $\Gamma$ when the structure is threedimensional. Other possibility to determine $J$-integral, the equation 2 can be used, but the strain fields (gradients) must be obtained [7]. These fields are extracted on the experimental plate and 3D numerical model only the free-surface. The present work studies the $J$-integral values obtained from 3D simulation and experimental test. The aims of this paper are also to determine if the 3D structure modify the strain fields near the crack tip, so the $J$-integrals evaluation. The second work is to define a distance where equation 1 is valid to determine the energy release rate in the real case. For the numerical simulation, finite element software is used (CASTEM). The calculation of experimental $J$ -

a e-mail : stephen.hedan@univ-poitiers.fr

This is an Open Access article distributed under the terms of the Creative Commons Attribution-Noncommercial License 3.0, which permits unrestricted use, distribution, and reproduction in any noncommercial medium, provided the original work is properly cited. 


\section{EPJ Web of Conferences}

integral requires experimental in-plane fields on the free surface and 2 theoretical formulations [8]. Experimental displacement fields $\mathrm{u}_{x-\exp }$ and $\mathrm{u}_{y-\exp }$ are measured in a SEN tensile specimen loaded in mode I (crack opening) using the grid method and are drifted. The choice of these formulations is emphasised by the presence of noise in the experimental data and by the necessity to derive the displacements. To calculate the $J$-integral using the numerical simulations, it is possible to use the G-theta procedure [9-10]. This procedure allows one value of $\mathrm{G}$ on the crack front to be extracted. In this paper, we want to calculate the energy release rate for different contours $\Gamma$ centred on the tip. $J$ integral in the linear elastic case is determined by the calculation of displacement gradients [11] and by the application of the following equation.

$$
\mathrm{J}_{\text {theo }}=\frac{\mathrm{K}_{1}^{2}}{\mathrm{E}}
$$

with $K_{I}=\sigma \sqrt{ }(\pi a) . Y(a / W)$ [7], E Young's modulus,

$$
\begin{aligned}
J=\frac{E}{4(1+v)} \int_{\Gamma}\left[\left\{k_{1}\left(\frac{\partial u_{y}}{\partial y}-\frac{\partial u_{x}}{\partial x}\right)\left(\frac{\partial u_{x}}{\partial x}+\frac{\partial u_{y}}{\partial y}\right)+\left(\frac{\partial u_{x}}{\partial y}+\frac{\partial u_{y}}{\partial x}\right)\left(\frac{\partial u_{x}}{\partial y}-\frac{\partial u_{y}}{\partial x}\right)\right\} d y\right. \\
\left.+2\left(\frac{\partial u_{x} \partial u_{x}}{\partial x \partial y}+k_{2} \frac{\partial u_{x} \partial u_{y}}{\partial x \partial x}+k_{2} \frac{\partial u_{y} \partial u_{y}}{\partial x \partial y}\right) d x\right]
\end{aligned}
$$

with: $v$ Poisson's ratio, $\partial \mathrm{u}_{i} / \partial i$ displacement gradients with $i=x, y$ and $\mathrm{k}_{1}=2 /(1-\mathrm{v}), \mathrm{k}_{2}=(1+\mathrm{v}) /(1-\mathrm{v})$ constants characterising the stress plane problem.

\section{Experimental setup}

Experimentally, it is difficult to have access to the displacement gradients on the free surface. Nevertheless, it is possible to derivate the displacement fields near the crack tip. An optical method has been adapted to extract the large fields in fracture mechanics [8]. The grid method has the advantage of giving an accurate measurement with a high spatial resolution. Thus it is consequently well-adapted to measure in the presence of singularities. The creation of a grating is achieved by evaporating aluminium in a vacuum chamber through a master grid. The advantage of this technique is to give rapidly a grid of a large size $\left(150 \times 150 \mathrm{~mm}^{2}\right)$ and with pitch sizes (Fig.1) of $50 \mu \mathrm{m}$ to few millimetres. The grid thus created allows the two components of in-plane displacement to be attained and is considered here.

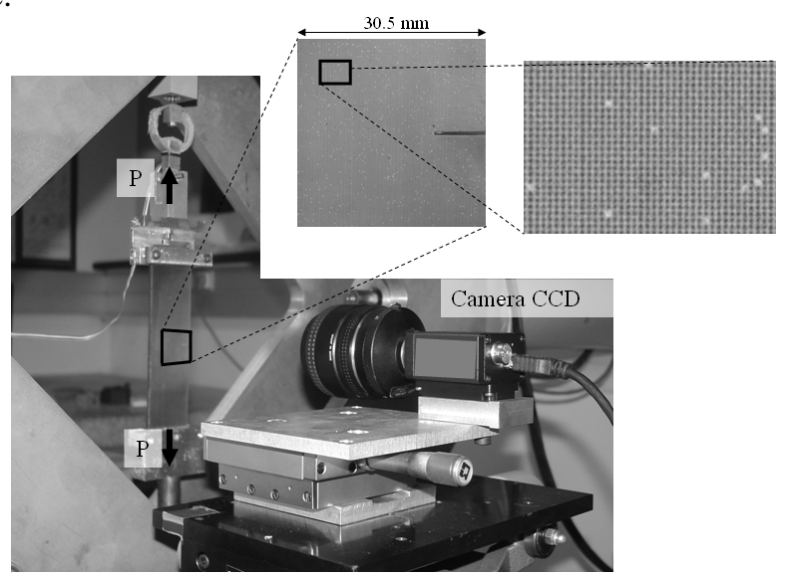

Fig. 1. Experimental set-up composed of a camera, a loading system and a cracked specimen covered by a grid 
Analysing grids to obtain the displacement fields consists in demodulating the image intensity I(x,y). In the current investigation a phase demodulation program using only one image was chosen. This referred to here as the MPC method [12] and gives an accuracy of p. $2 \tilde{\pi} / 40$. The first goal is to extract $\phi_{l-x}(x, y)$ and $\phi_{l-y}(x, y)$ ( $l$ : loading state) from a single fringe pattern and this is then extended to the analysis of the grids. This method based on a correlation technique, searches for the best degree of similarity between a mathematical model and a real grid pattern. $\left(\phi_{l-x}(x, y)\right.$ and $\left.\phi_{l-y}(x, y)\right)$ are obtained and correspond to the 2 directions. The wrapped phases can easily be unwrapped using a classical algorithm [13]. The experimental in-plane displacement fields $\mathrm{u}_{x_{-} \exp }$ and $\mathrm{u}_{y_{-} \exp }$ (Fig.2) can be expressed by the following equations, with an origin of coordinates $(x, y)$ being placed at the crack tip.

$$
\begin{aligned}
& \mathrm{u}_{x_{-} e x p}(x, y)=\frac{\mathrm{p}}{2 \pi} \cdot\left(\phi_{l-x}(x, y)-\phi_{0-x}(x, y)\right) \\
& \mathrm{u}_{y_{-} e x p}(x, y)=\frac{\mathrm{p}}{2 \pi} \cdot\left(\phi_{l-y}(x, y)-\phi_{0-y}(x, y)\right)
\end{aligned}
$$

with p: grid pitch $(=50 \mu \mathrm{m})$

Experiments are performed on a $6 \mathrm{~mm}$ thick SEN specimen (PMMA) as shown in Figure 1. The mechanical characteristics are obtained by optical device named mark tracking method [14] and geometrical dimensions ( $\mathrm{W}=$ width), ( $\mathrm{L}=$ length) and ( $\mathrm{h}=$ thickness) of specimen are listed in table 1.

Table 1. Material properties and geometrical dimensions of the PMMA specimen.

\begin{tabular}{|c|c|c|c|c|c|c|}
\hline Materials & $\mathrm{a}[\mathrm{mm}]$ & $\mathrm{W}[\mathrm{mm}]$ & $\mathrm{L}[\mathrm{mm}]$ & $\mathrm{h}[\mathrm{mm}]$ & $\mathrm{E}[\mathrm{MPa}]$ & $v$ \\
\hline PMMA & $9 \pm 0.1$ & $40 \pm 0.2$ & $145 \pm 0.2$ & $6 \pm 0.1$ & $2825 \pm 25$ & $0.4 \pm 0.04$ \\
\hline
\end{tabular}

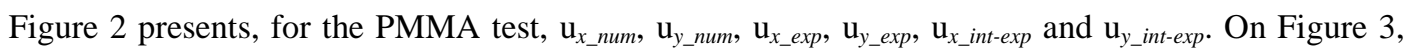
the plots of $\mathrm{u}_{x_{-} \text {exp }}$ and $\mathrm{u}_{y_{-} \text {exp }}$ in 2 directions $(\theta= \pm 45$ and \pm 135 degrees $)$ are presented for the PMMA test and the presence of a significant noise can be noted. The presence of the latter in the experimental in-plane fields not allows displacement gradients to be directly measured. The used approach consists to minimize the displacement gaps by 2 adapted formulations (eq.5 and eq.6) representing correctly the displacement fields and the displacement gradients. These expressions are based on the Arakawa's formulations [15] and are modified by Hedan [8].

$$
\begin{gathered}
\mathrm{u}_{x_{-} \text {int }-\exp }(r, \theta)=- \text { v.c.r. } \cdot \cos \theta+C_{1 x} \cdot \cos \left(\frac{\theta}{2}\right)-C_{2 x} \cdot \cos \left(\frac{3 \theta}{2}\right) \cdot a \cdot \varepsilon \cdot\left(1-\mathrm{e}^{-\lambda_{x}\left(\frac{r}{\mathrm{a}}\right)^{\alpha_{x}}}\right) \\
\mathrm{u}_{y_{-} \text {int }-\exp }(r, \theta)=\varepsilon \cdot r \cdot \sin \theta+C_{y} \cdot \sin ^{3}\left(\frac{\theta}{2}\right) \cdot a \cdot \varepsilon \cdot\left(1-\mathrm{e}^{-\lambda_{y}\left(\frac{r}{\mathrm{a}}\right)^{\alpha_{y}}}\right)
\end{gathered}
$$

The values of 7 constants (Table 2) are obtained using a least squares method. It is seen that the developed formulations are in good agreement with the experimental displacement fields (Figure 2). 


\section{EPJ Web of Conferences}

Table 2. Results of 7 constants after minimization with experimental displacements.

\begin{tabular}{|c|c|c|c|c|}
\hline Displacements & \multicolumn{4}{|c|}{ Experimental data } \\
\hline Horizontal & $\mathrm{C}_{I x}$ & $\mathrm{C}_{2 x}$ & $\lambda_{x}$ & $\alpha_{x}$ \\
\cline { 2 - 5 } $\mathrm{u}_{\text {I_int-exp }}$ & 11.7 & 4.7 & 0.1 & 0.475 \\
\hline Vertical & \multicolumn{2}{|c|}{$\mathrm{C}_{y}$} & $\lambda_{y}$ & $\alpha_{y}$ \\
\cline { 2 - 5 } $\mathrm{u}_{\text {__int-exp }}$ & \multicolumn{2}{|c|}{3.0} & 1.7 & 0.6 \\
\hline
\end{tabular}

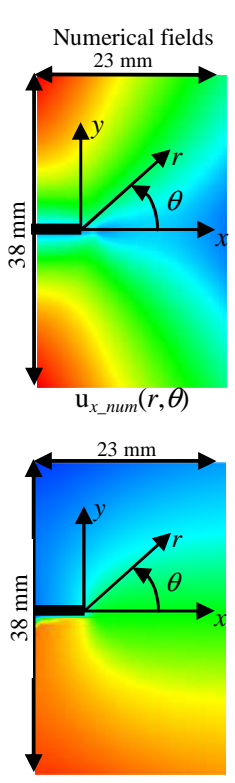

Experimental fields

Calculated from

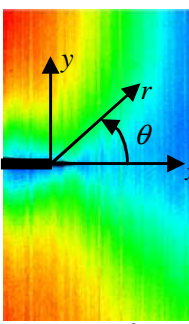

$\mathrm{u}_{x \in e x p}(r, \theta)$

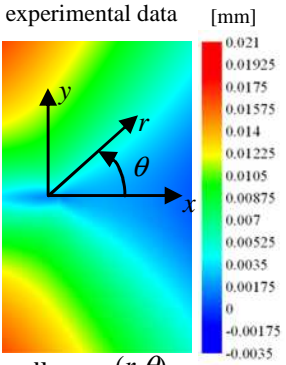

$\mathrm{u}_{y_{\_} \text {num }}(r, \theta)$

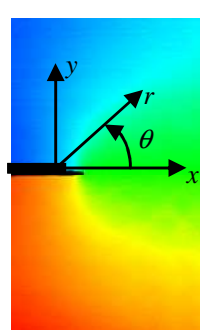

$\mathrm{u}_{y_{-} e x p}(r, \theta)$

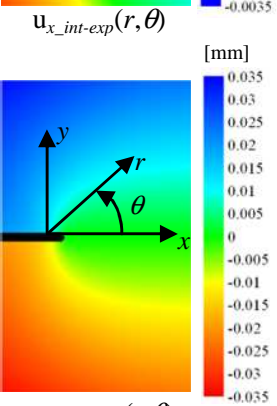

$\mathrm{u}_{y_{-} \text {int-exp }}(r, \theta)$

Fig. 2. In-plane displacement fields obtained by the FEA method, measured with the grid method and obtained by minimization.

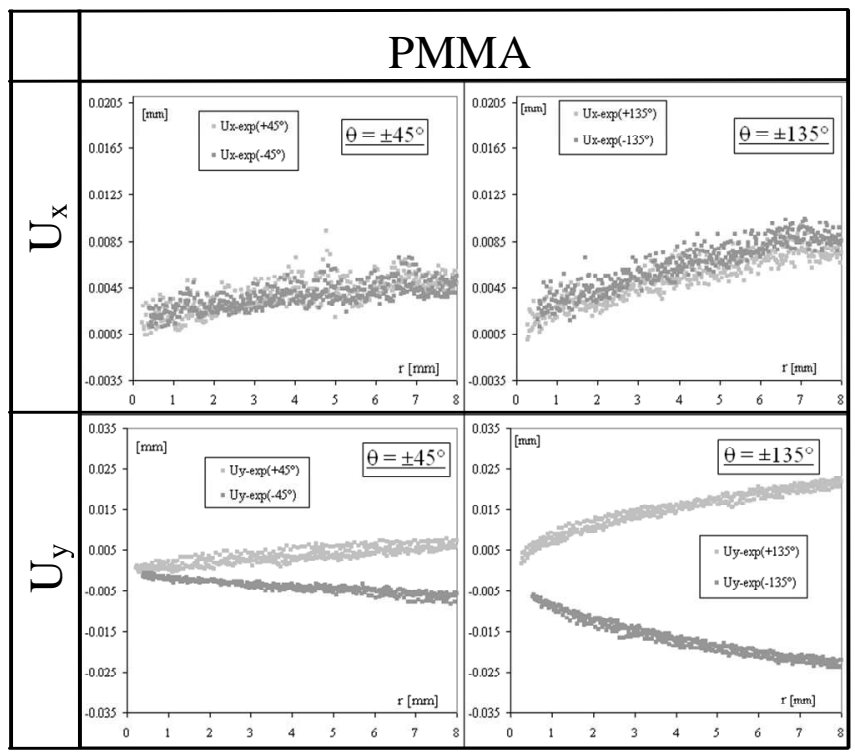

Fig. 3. Experimental displacements for the PMMA specimen in the $x$ and $y$ directions for $\theta=45$ and $135^{\circ}$ 


\section{Finite element analysis}

A finite element analysis of the SEN specimen is undertaken in order to estimate gradient displacements employing the CASTEM finite element program. This software is developed by the French atomic energy commission (CEA). A half of a specimen is modelled and analysed. This 3D model is composed of 8-node trilinear hexahedron elements and of a similar circular mesh surrounding the crack tip. A circular mesh centred on the crack tip is used and is based on the works of Nakamura [4], with the radius equal to $\mathrm{a}=9 \mathrm{~mm}$. To accommodate the variation of the stress in the thickness of the plate, the successive element layers are gradually reduced as far as the free surface (Figure 3). A quarter of a specimen is modelled. This representation has allowed the state of three-dimensional stresses near the singular zone to be characterised. The numerical specimen is modelled on the geometric dimensions, (Table 1). The SEN specimen is loaded in mode I (crack opening mode) and the used brittle material (PMMA) presents the mechanical characteristics listed in the table 1.

The 3D character, the boundary conditions and behaviour law (linear elastic) are validated by comparisons of experimental displacement fields on the free surface [8] and by comparisons displacement fields on the bulk of 3D real cracked specimen [16].

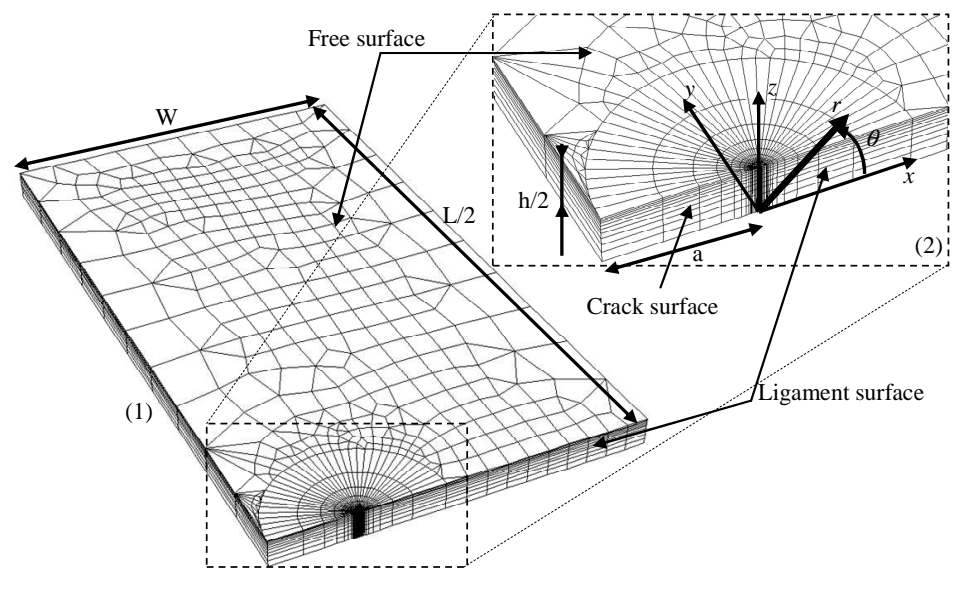

Fig. 3. Finite element meshes in the full model (1) and near-crack-tip region (2).

The Grad procedure of CASTEM software allows the displacement gradients on the nodes to be extracted. To calculate the $J$-integral for circular contours $\Gamma$ with varying dimensions, a $\mathrm{C}++$ program is written using a form of the $J$-integral that is expressed in terms of the displacement gradients and material constants (equation 1). In Fig. 4, the $J$-plots versus to (r) are made.

\section{J-integral calculations}

It was possible to calculate the experimental $J$-integral $\left(\mathrm{J}_{\text {int/exp }}\right)$ and to compare these values with the other $J$-integrals obtained by numerical simulation $\left(\mathrm{J}_{\text {num }}\right)$ and by $2 \mathrm{D}$ model $\left(\mathrm{J}_{\text {theo }}\right)$. The $\mathrm{J}$-integral calculation is limited to a maximal radius equal to the crack length (a), because a circular contour centred in the crack tip is used. 


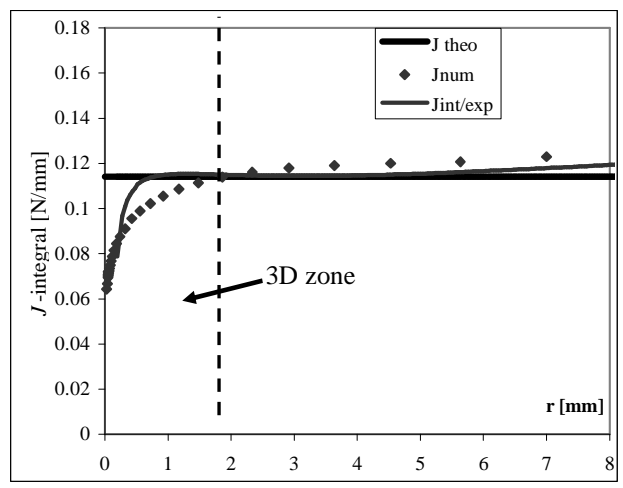

Fig. 4. Plot of J-integrals calculated from the theoretical formulations, from the numerical gradients and from the interpolation of the experimental displacement.

On Figure 4, the observed gap between $\mathrm{J}_{\text {num }}$ and $\mathrm{J}_{\text {theo }}$ is localized at the neighbourhood of the crack tip $(\mathrm{r}<2 \mathrm{~mm})(\mathrm{R}<0.33) . \mathrm{J}_{\text {theo }}$ is sufficient to determine $\mathrm{G}$, when $\mathrm{r}>2 \mathrm{~mm}$ and $J$-integrals are independent to $\Gamma$. The differences between the $\mathrm{J}_{\text {int-exp }}$ and $\mathrm{J}_{\text {num }}$ are due to the amplitude of noise versus to the amplitude of experimental displacements (Figure 3). The noise has an influence on the values of 7 constants (eq. 5-6) and then on the values of displacement gradients and $\mathrm{J}_{\text {int_exp }}$.

One can also see that $J_{\text {intexp }}$ and $J_{\text {num }}$ decrease when $r>0$ and $r<2 \mathrm{~mm}(R=r / h<0.333)$. This can be explained by the presence of local $3 \mathrm{D}$ effects in the experimental test and the numerical simulation, classically observed in literature [2-4] and called 3D zone.

\section{Conclusions}

The comparison of $\mathbf{J}_{\text {num }}$ and $\mathbf{J}_{\text {int/exp }}$ allow the gradient fields to be obtained with a good accuracy even if the experimental in-plane displacement fields are noised. Experimental and numerical results show and confirm a J-integral dependence in comparison to $\Gamma$ for a 3D structure. When a radius $r>2 \mathrm{~mm}$, $\mathrm{J}_{\text {theo, }}$, developed for two-dimensional conditions, is sufficient to determine the energy release rate of the $3 \mathrm{D}$ cases. By these experimental and numerical studies, the $J$-integral calculation confirms a presence of 3D effects surrounding the tip, as observed by out-of-plane displacement measurements, or numerical stress fields. For $r<2 \mathrm{~mm}$, the surface measurements are not sufficient to estimate the energy release rate $\mathrm{G}$ and consequently it is necessary to have a $3 \mathrm{D}$ measurement on the bulk of cracked specimen.

\section{References}

1. J. R. Rice, J. Appl. Mech., 379-386 (1968).

2. A. J. Rosakis, K. Ravi-Chandar, Int. J. Sol. Struct. 22(2), (1986).

3. R. Pfaff, P. Washabaugh, , W. Knauss, , Int. J. Sol. Struct. 32(6-7), (1995).

4. T Nakamura, D M Parks, J. Appl. Mech. 55, (1988).

5. H. M. Westergaard, J. Appl. Mech., (1939).

6. P.-F. Luo, C.-H. Wang, Strain, 44(3), (2008).

7. I Barsoum, K. S. Ravi Chandran, Engng Fract Mech, 70, (2003).

8. S. Hedan, V.Valle, M. Cottron, Accepted Exp Mech. 50, (2010).

9. T. K. Hellen., Int J Num Meth Eng, 9, (1975).

10. F. Dubois, C. Chazal, C. Petit., Mech Time Depend Mater, 2(3), (1998).

11. Y. L. Kang, Z. F. Zhang, H. W. Wang, Q. H. Qin, Mat. Sc. Engng A, 394 (1-2), (2005).

12. E. Robin E, V. Valle, F. Bremand, J. Appl. Opt., 44(34), (2005). 
13. F. Bremand, Opt. Lasers Eng. 21, (1994).

14. N. Bretagne, V. Valle, J.C. Dupre, NDT \& E, 38(4), (2005).

15. K. Arakawa, K. Takahashi, Int. J. Fract. 86(4), (1997).

16. Y. Barranger, P. Doumalin, J-C. Dupre, A. Germaneau, S. Hedan, V. Valle, Engng Fract. Mech., 76(15), (2009). 